\title{
Topology-Preserving Discrete Deformable Model: Application to Multi-segmentation of Brain MRI
}

\author{
Sanae Miri ${ }^{1,2}$, Nicolas Passat ${ }^{1}$, and Jean-Paul Armspach ${ }^{2}$ \\ ${ }^{1}$ LSIIT, UMR 7005 CNRS/ULP, Strasbourg 1 University, France \\ ${ }^{2}$ LINC, UMR 7191 CNRS/ULP, Strasbourg 1 University, France
}

\begin{abstract}
Among the numerous 3D medical image segmentation methods proposed in the literature, very few have intended to provide topologically satisfying results, a fortiori for multiple object segmentation. In this paper, we present a method devoted to parallel segmentation of the main classes of cerebral tissues from 3D magnetic resonance imaging data. This method is based on a multi-class discrete deformable model strategy, starting from a topologically correct model, and guiding its evolution in a topology-preserving fashion. Validations on a commonly used cerebral image database provide promising results and justify the further development of a general methodological framework based on the concepts exposed in this preliminary work.
\end{abstract}

Keywords: Topology preservation, multi-segmentation, discrete deformable model, medical imaging.

\section{Introduction}

When performed on medical data, segmentation consists in decomposing the image support into two sets: the searched structure, and the "useless" part of the image, usually by using photometric and/or shape knowledge related to the anatomical elements and the image acquisition process. However complex organs, such as the brain, require more sophisticated segmentation strategies. Indeed, the brain is composed of several different tissues and anatomical structures presenting non-trivial shapes and organised in a complex fashion. Consequently, in order to provide results enabling to facilitate the analysis of brain images, segmentation methods have to correctly divide such images into $k>2$ classes corresponding to the several anatomical and pathological elements visualised. The determination of multiple classes, and the complex shape and organisation of brain structures require to base the developed strategies not only on "classical" intensity and shape hypotheses, but also on more sophisticated geometrical, relational and topological ones. In this context, the notion of deformable model can deal with these different kinds of a priori anatomical knowledge, and then lead to the creation of efficient tools.

In the sequel, a method devoted to brain structure segmentation from 3D magnetic resonance imaging $(\mathrm{MRI})$ data is described. It relies on a multi-class 
discrete deformable model strategy, starting from a topologically-correct model, and evolving without topology modifications until segmenting the brain into four classes corresponding to the four main intracranial structures. This method constitutes a preliminary attempt to illustrate and validate the potential efficiency of a general methodology consisting in developing complex - and realistic - anatomical models of the brain and deforming them under high-level a priori anatomical knowledge to obtain accurate segmentation results.

\section{Related Work}

In 810, some strategies are described for vascular networks segmentation. Based on the assumption that these networks are organised as tree structures, they propose to monotonically modify a simply connected model by addition (or subtraction) of simple points 2. Using the same idea, brain surface segmentation methods 6]5] use the assumption that the cortex is a topological hollow sphere. They thicken/thin a subset/superset corresponding to the white matter/intracranial volume to finally detect the cortex/cortical surface. Topology preserving is handled by considering simple point [6] or complexes [5].

The first methods devoted to sequential multi-segmentation of $3 \mathrm{D}$ brain images are described in 977. They segment cerebral anatomical structures in a coarse to fine way, the result of each segmentation step enabling to initialise the next one. Recently, the first parallel cerebral structure multi-segmentation method [1] has been proposed. Based on a topological model of the brain, it iteratively performs a classification/homotopic skeletonisation/homotopic thickening process. The initialisation requires a rigid-registration of a precomputed topological model which has to present structures sufficiently large not to be topologically altered by the deformation field. Moreover, the issue of modelling and preserving the topology of a non-binary image is not clearly considered (all classes are handled in a 6 -adjacency framework, and some voxels may be unclassified). Finally, the iteration of numerous steps is computationally quite expensive.

As in [1, the work described hereafter is based on a topological model of the brain structures which evolves, thanks to the notion of simple point, in a topology preserving fashion. However, it presents several major differences. The topological model is more simple than the one of [1] (both still being non-anatomically compliant) but it enables to correctly model and preserves topological properties during the deformation process. The deformation algorithm is not based on successive monotonic processes, but on a smooth non-monotonic one, leading to a low computation time method. Finally, this work constitutes a preliminary study devoted to assess the potential efficiency of the proposed methodology.

\section{Background}

\subsection{Anatomical and Physical Notions}

Brain Anatomy. The brain is mainly composed of three kinds of tissues: grey matter, white matter and cerebrospinal fluid. It is constituted of several structures 
located in the intracranial volume: brainstem, cerebellum, ventricles, cortex, etc. Most of them have complex shapes, but are quite invariant from one brain to another, particularly from topological and relational points of view. In a macroscopic and simplified way, the brain is surrounded by a layer of tissues and cerebrospinal fluid. Except on its inferior face (where are located the cerebellum and the brainstem), the external surface of the brain is composed of the cortex, which is a thick convoluted ribbon of grey-matter. Under this cortical surface, white matter surrounds the ventricles, which are cavities containing cerebrospinal fluid, linked together by thin tunnels.

Magnetic Resonance Imaging. Magnetic resonance imaging (MRI) is a class of medical image acquisition protocols taking advantage of the magnetic properties of hydrogen atoms located in living tissues to visualise internal anatomical structures. There exist several modalities of MRI, each one presenting specific properties. The most commonly used for brain visualisation is T1 MRI, enabling to discriminate the white matter, the grey matter and the cerebrospinal fluid, which present decreasing and globally homogeneous intensities in such data. An example of T1 MRI axial slice is illustrated in the 1st picture of Fig. 1, where we can observe three classes of intensity corresponding to the three classes of tissues previously enumerated.

\subsection{Notations and Hypotheses}

Notations In the sequel, the grey matter, white matter, and cerebrospinal fluid will be denoted by GM, WM, and CSF, respectively. A 3D MRI data will be considered as a function $I: E \rightarrow \mathbb{N}$ (a MRI data having discrete positive values), where $E$ is the support of $I$, and has the form $\left[0, d_{x}-1\right] \times\left[0, d_{y}-1\right] \times\left[0, d_{z}-1\right]$, $d_{x}, d_{y}, d_{z}$, being the dimensions of $I$ (generally 256, for brain millimetric data).

Hypotheses. The purpose of this work is the evaluation of the potential efficiency of parallel segmentation of the different cerebral structures, based on a discrete topology-guided deformable model process. In order to lead to a final application which will be fully efficient, such a strategy will require to deal with several issues, some of them still being open problems (this will be discussed in Section (6). To be able to evaluate the feasibility and efficiency of this strategy before dealing with these issues, we chose to made two main simplifying assumptions: (1) the three different tissues constituting the brain present a quite homogeneous signal, i.e. MRI noise and signal distortion are low, and (2) the brain presents a relational and topological structure more simple than the real one (by omission of the smallest structures), i.e. the brain will be considered as composed of four "tissue layers" hierarchically surrounded by each others. It has to be noticed that these simplifying hypotheses do not alter the general segmentation methodological framework which will be illustrated hereafter. The first hypothesis will enable to provide a simple constraint to deform the topological model during the segmentation process, while the second one will allow the development of a tractable multi-class topological model. 

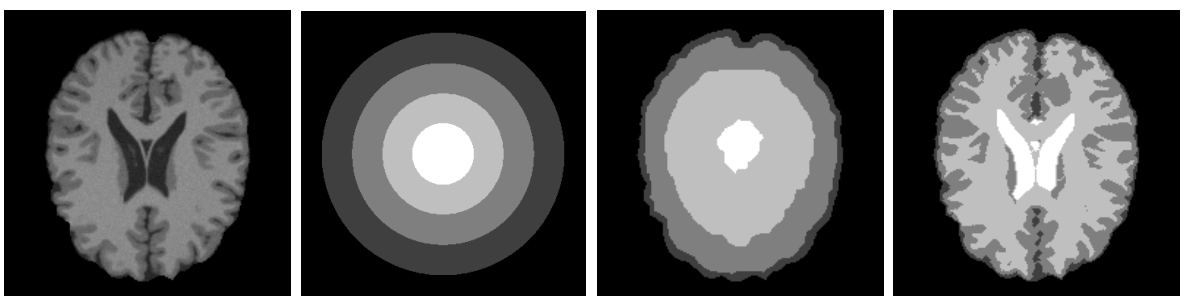

Fig. 1. From left to right: axial slice of a T1 MRI restricted to the intracranial volume $\left(I_{\mid E^{\prime}}\right)$; topological model (from white to dark grey: $C_{v}^{i}, C_{w}^{i}, C_{g}^{i}$, and $C_{s}^{i}$ ); initialisation of the segmentation process of the MRI $\left(C^{i}\right)$; expected result of the segmentation of the MRI (from white to dark grey: $C_{v}^{i}, C_{w}^{i}, C_{g}^{i}$, and $C_{s}^{i}$ )

\section{Method}

\subsection{Input/Output}

The method takes as input a T1 MRI of the brain, $I: E \rightarrow \mathbb{N}$, from which the intracranial volume $E^{\prime} \subset E$ has been extracted 1 (Fig. 1, 1st picture), and two threshold values $\mu_{1}<\mu_{2} \in \mathbb{N}$ delimiting the $\mathrm{T} 1$ signal intensity between $\mathrm{CSF} / \mathrm{GM}$, and GM/WM, respectively. The method output is a partition $C=$ $\left\{C_{s}, C_{g}, C_{w}, C_{v}\right\}$ of $E^{\prime}$, where $C_{s}, C_{g}, C_{w}$, and $C_{v}$ correspond to the sulcal (i.e. external) CSF, GM, WM, and ventricular (i.e. internal) CSF classes, respectively (Fig. 1, 4th picture).

\subsection{Initialisation}

The segmentation process has to be initialised from a topological model $C^{i}$ of $E^{\prime}$ having the desired topology: $C_{v}^{i}$ is simply connected (1 connected component, 0 hole, 0 cavity), and successively surrounded by $C_{w}^{i}, C_{g}^{i}$, and $C_{s}^{i}$ which are topological hollow spheres (1 connected component, 0 hole, 1 cavity), hierarchically organised, as illustrated in a 2D fashion in Fig. 1 (2nd picture).

In order to generate this initial model, a distance map is computed from $E^{\prime}$. This discrete map (providing the chamfer distance of each point of $E^{\prime}$ to its border, i.e. to the brain hull) is then used to guide a homotopic reduction process. Simple points are iteratively removed from $E^{\prime}$, with a highest priority to the points being the closest from the border of $E^{\prime}$. These removals are performed until reaching a distance $d_{v}>0$ corresponding to an approximation of the sulcal CSF thickness (which can be expressed as a ratio of the highest distance of the map). The set composed by the removed points (necessarily presenting a hollow sphere topology) corresponds to the sulcal CSF class $C_{v}^{i}$, while the remaining set of $E^{\prime}$ corresponds to $C_{w}^{i}, C_{g}^{i}$, and $C_{s}^{i}$. The same process is iterated on this

\footnotetext{
${ }^{1}$ Justified by the existence of efficient tools, the preliminary extraction of the intracranial volume is a frequent preprocessing procedure before cerebral structure segmentation $5[6[4]$.
} 
remaining set, with distances $d_{g}, d_{w}>0$, to successively generate $C_{g}^{i}(\mathrm{GM})$ and $C_{w}^{i}(\mathrm{WM})$ which, by construction, also present a hollow sphere topology. The finally remaining component, thus presenting a simply-connected topology, corresponds to the "central" part of the model, i.e. $C_{s}^{i}$ (ventricular CSF). A 2D axial slice of an example of initial model is illustrated in Fig. 11 (2nd picture).

In $\mathbb{Z}^{3}$, the use of such a model, organised as a set of "hierarchically included" connected components, implies to choose dual adjacencies for these successive components (i.e. classes). The 6-adjacency has been considered for $C_{g}^{i}$ (and thus $\left.C_{v}^{i}\right)$, since the GM is geometrically organised as a "thick" ribbon, while the 26adjacency has been considered for $C_{w}^{i}, C_{v}^{i}$, since they both present "thin" details near the cortex.

From a topological point of view, $C^{i}$, although composed of four distinct classes, can be considered as a binary image made of an object $X=C_{s}^{i} \cup C_{w}^{i}$ and of the background $\bar{X}=C_{g}^{i} \cup C_{v}^{i}$, in a $(26,6)$-adjacency framework. This is justified by the fact that each class is adjacent to - at most - two other classes (the surrounded and surrounding ones), which are not adjacent with each other. Both adjacent classes then locally correspond to the "background" of this class, considered as a binary structure.

\subsection{Discrete Deformable Model}

The discrete deformable model, which constitutes the main part of the method, consists in "deforming" the four classes without altering their topology until reaching the segmentation of the searched structures. This deformation is performed by modifying the frontiers between the classes, which is actually equivalent to modify the frontier between the sets $X$ and $\bar{X}$. The - topology preserving - evolution of the frontier of a binary object can be performed by adding/removing simple points to/from this object. Adding/removing a point to/from $X$ is equivalent to add/remove a point to/from $C_{s}^{i}$ or $C_{w}^{i}$ while removing/adding it from/to $C_{g}^{i}$ or $C_{s}^{i}$. For a given point, the binary growth/reduction of $X$ then corresponds to a reclassification (class modification between two "adjacent" classes) in $C^{i}$. It has to be noticed that, given the chosen dual adjacencies for the successive classes, a simple point of $X$ or $\bar{X}$ is only adjacent to one connected component of $X$ and one connected component of $\bar{X}$, i.e., only adjacent to its own class and the class where it could be reclassified. Consequently, (1) such points are located at the frontier between two classes, and (2) there is no ambiguity regarding their potential reclassification.

The proposed deformation model aims at iteratively reclassifying the points located at the frontiers between classes, making all these frontiers parallely evolving in a smooth and non-monotonic way. The deformation is guided by photometric constraints. A cost is provided for each point $x \in E^{\prime}$ : if the grey-level value $I(x)$ of $x$ is not coherent w.r.t. the expected value interval (provided by the input threshold values $\mu_{1}$ and $\mu_{2}$ ) of the class it belongs to, the - positive - distance between $I(x)$ and this interval is assigned as cost for $x$. The deformation model iteratively switches "misclassified" simple points from one class to another, giving the highest priority to the "most misclassified" ones (i.e. those having the 


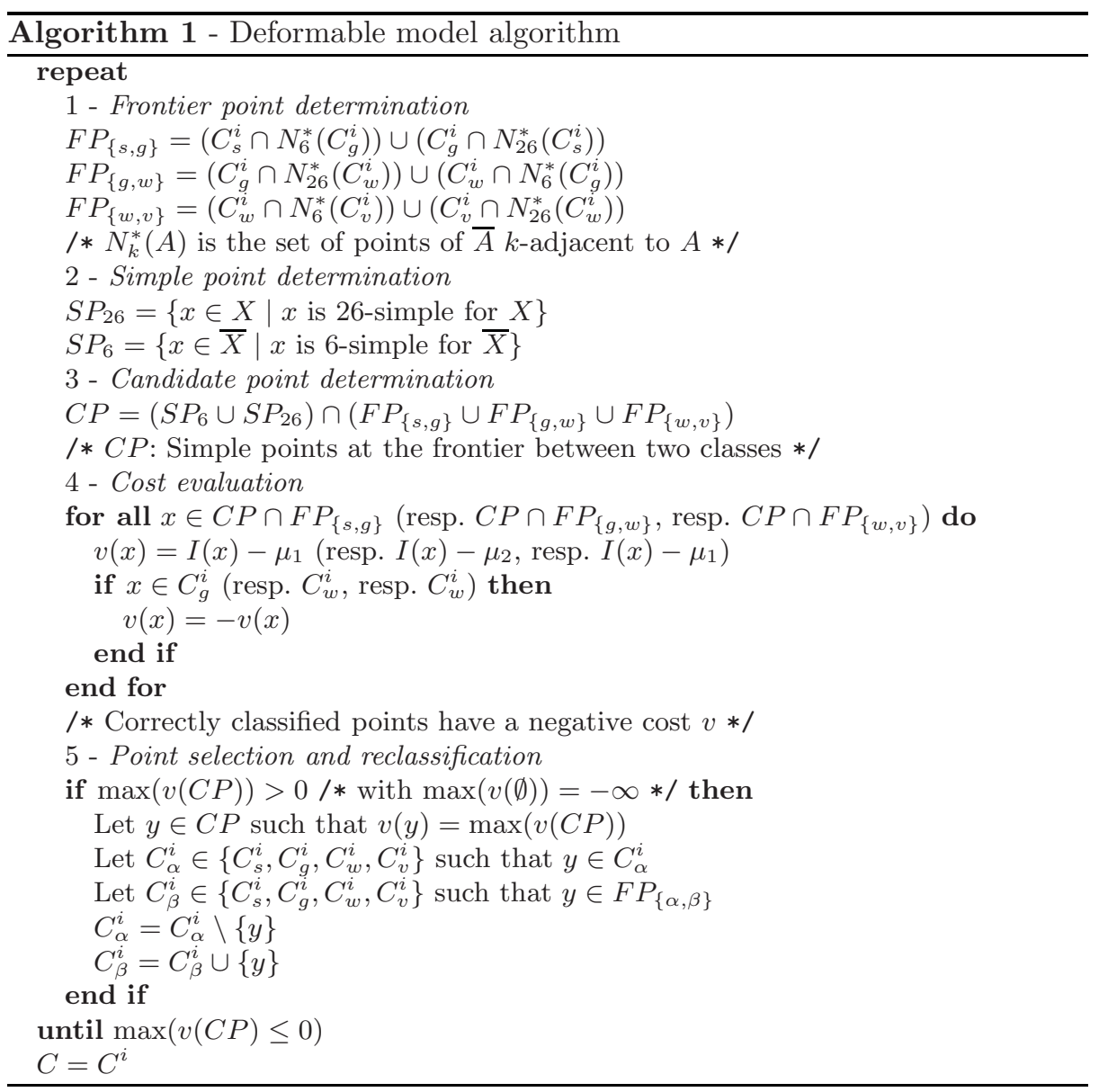

highest cost), until no simple point or no misclassified point is detected. This process, informally explained here, is clearly detailed in Alg. 1

\section{Experiments and Results}

\subsection{Complexity and Computation Time}

For simplicity's sake, the segmentation algorithm detailed in Alg. 1 is presented in a "non-optimal" fashion, using set-based notations. However, it was implemented using efficient data structures and strategies (based on ordered FIFO lists), enabling to reach its optimal - linear - algorithmic complexity $O\left(\left|E^{\prime}\right|\right)$.

The proposed method has been implemented in $\mathrm{C}++$, and integrated into the multidimensional medical image processing and analysis software platform developed by the LINC. The tests and validations were performed on a $3 \mathrm{GHz}$ processor / 2 GB memory personal computer. In such conditions the computation time for processing a $256^{3} 3 \mathrm{D}$ T1 MRI vary between 1 and 2 minutes, 

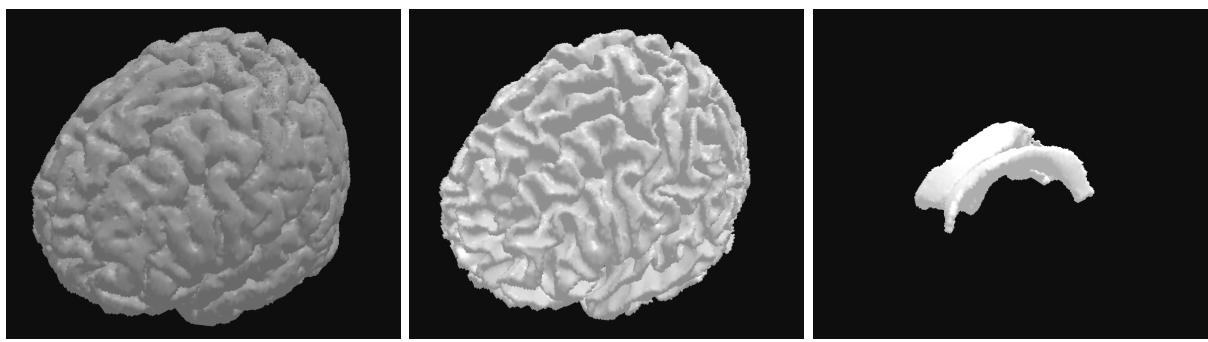

Fig. 2. 3D visualisation of brain segmentation results provided by the proposed method. From left to right: $C_{g}(\mathrm{GM}), C_{w}(\mathrm{WM})$, and $C_{v}$ (ventricular CSF).

Table 1. Sensitivity, specificity and similarity measures of segmentation results on the BrainWeb database, for various noise ratios

\begin{tabular}{|c|c|c|c|c|c|c|c|c|c|}
\hline \multirow{2}{*}{ Noise } & \multicolumn{3}{|c|}{ Sensitivity } & \multicolumn{3}{c|}{ Specificity } & \multicolumn{3}{c|}{ Similarity } \\
\cline { 2 - 10 } & CSF & WM & GM & CSF & WM & GM & CSF & WM & GM \\
\hline $0 \%$ & $88.5 \%$ & $99.4 \%$ & $81.5 \%$ & $99.4 \%$ & $98.2 \%$ & $99.8 \%$ & $83.6 \%$ & $91.8 \%$ & $89.0 \%$ \\
\hline $1 \%$ & $88.9 \%$ & $99.3 \%$ & $83.2 \%$ & $99.3 \%$ & $98.5 \%$ & $99.8 \%$ & $83.2 \%$ & $93.1 \%$ & $90.0 \%$ \\
\hline $3 \%$ & $89.7 \%$ & $95.4 \%$ & $85.9 \%$ & $98.9 \%$ & $99.6 \%$ & $99.4 \%$ & $79.8 \%$ & $95.7 \%$ & $90.4 \%$ \\
\hline $5 \%$ & $89.8 \%$ & $87.2 \%$ & $81.2 \%$ & $98.6 \%$ & $99.7 \%$ & $98.5 \%$ & $76.4 \%$ & $91.6 \%$ & $84.8 \%$ \\
\hline $7 \%$ & $90.0 \%$ & $80.0 \%$ & $75.9 \%$ & $98.3 \%$ & $99.6 \%$ & $97.7 \%$ & $74.0 \%$ & $87.1 \%$ & $79.2 \%$ \\
\hline $9 \%$ & $82.3 \%$ & $62.1 \%$ & $66.9 \%$ & $97.7 \%$ & $99.6 \%$ & $95.7 \%$ & $65.6 \%$ & $75.0 \%$ & $68.1 \%$ \\
\hline
\end{tabular}

depending on the quality of the data. This relatively low computation time, associated to the automation of the process, results in an easy use of the method for clinicians.

\subsection{Validations}

In order to quantitatively and qualitatively validate the method, T1 MRI data provided by the commonly used BrainWeb2 database have been considered.

Results obtained for images corresponding to noise ratios from $0 \%$ to $9 \%$ of corrupted voxels have been analysed by considering, for each class of tissue 3 , three statistical measures: sensitivity $(t p /(t p+f n))$, specificity $(t n /(t n+f p))$ and similarity $(2 . t p /(2 . t p+f p+f n))$, with $t n, t p, f n$ and $f p$ being the number of true negative, true positive, false negative and false positive voxels, respectively. These measures are summarised in Table 1 The result illustrated in Fig. 2 corresponds to the first line of this table.

From a quantitative point of view, the obtained results are still not perfect. For non-noisy data, they are similar to results proposed by other kinds of methods, such as the statistical one developed in 4]. For noisy data, the results are

\footnotetext{
${ }^{2}$ http://www.bic.mni.mcgill.ca/brainweb

${ }^{3}$ The classes $C_{s}$ and $C_{v}$, both corresponding to CSF, have been fused, since the BrainWeb ground truth does not discriminate them.
} 
less satisfactory, since the proposed method - in its primary version proposed here - strongly relies on photometric constraints. From a qualitative point of view, the results are, however, much better than those obtained from [4], and more generally from methods which do not rely on anatomical (i.e. geometrical, topological and/or relational) a priori knowledge. As an example, the cortex presents here a real thick surface, while the ventricular CSF is fully surrounded by the grey matter.

\section{Conclusion}

This method and encouraging results exposed in this paper, constitute preliminary works related to the parallel topology-preserving segmentation of structures from medical data, in a discrete deformation model framework. This field of research has not been extensively studied until now, probably because it requires to find correct solutions to non-trivial theoretical and practical issues. Here, a simplifying hypothesis enabled to consider a four label image of the brain as a binary one. The real topology of brain structures will not allow to keep considering such an hypothesis. The most important issue is then the determination of satisfying solutions for defining and modelling the topology of digital images composed of more than two classes of labels, and to deform such images while leaving unchanged their topological properties. Another way for further research deals with the determination of more sophisticated constraints for guiding the deformation process. Here, photometric parameters were considered. Other kinds of a priori anatomical knowledge [3] should be involved in the guidance of the deformation process, in order to make it as reliable as a human expert. The main challenges will consist in determining and formalising them in a way finally enabling to obtain a method being robust, while not excessively altering its algorithmic complexity and computation time.

\section{References}

1. Bazin, P.-L., Pham, D.L.: Topology-preserving tissue classification of magnetic resonance brain images. IEEE Transactions on Medical Imaging 26(4), 487-496 (2007)

2. Bertrand, G., Malandain, G.: A new characterization of three-dimensional simple points. Pattern Recognition Letters 15(2), 169-175 (1994)

3. Bosc, M., Heitz, F., Armspach, J.-P.: Statistical atlas-based sub-voxel segmentation of 3D brain MRI. In: Proceedings of International Conference on Image Processing - ICIP 2003. 10th International Conference, Barcelona, Spain, September 14-17 2003, vol. 2, pp. 1077-1080. IEEE Signal Processing Society, Los Alamitos (2003)

4. Bricq, S., Collet, C., Armspach, J.-P.: Triplet Markov chain for 3D MRI brain segmentation using a probabilistic atlas. In: Proceedings of International Symposium on Biomedical Imaging - ISBI 2006. 3rd International Symposium, Arlington, VA, USA, April 6-9 2006, pp. 386-389 (2006) 
5. Cointepas, Y., Bloch, I., Garnero, L.: Joined segmentation of cortical surface and brain volume in MRI using a homotopic deformable cellular model. In: Proceedings of 3D Digital Imaging and Modeling - 3DIM 1999, 2nd International Conference, Ottawa, Canada, October 4-8 1999, pp. 240-248 (1999)

6. Daragon, X., Couprie, M.: Neo-cortex segmentation from IRM data in order topology. In: Proceedings of Reconnaissance de Formes et Intelligence Artificielle - RFIA 2002, 13th Francophone Conference, Angers, France, January 8-10, 2002, vol. 3, pp. 809-818 (2002)

7. Dokládal, P., Bloch, I., Couprie, M., Ruijters, D., Urtasun, R., Garnero, L.: Topologically controlled segmentation of 3D magnetic resonance images of the head by using morphological operators. Pattern Recognition 36(10), 2463-2478 (2003)

8. Dokládal, P., Lohou, C., Perroton, L., Bertrand, G.: Liver blood vessels extraction by a 3-D topological approach. In: Taylor, C., Colchester, A. (eds.) MICCAI 1999. LNCS, vol. 1679, pp. 98-105. Springer, Heidelberg (1999)

9. Mangin, J.-F., Frouin, V., Bloch, I., Régis, J., López-Krahe, J.: From 3D magnetic resonance images to structural representations of the cortex topography using topology preserving deformations. Journal of Mathematical Imaging and Vision 5(4), 297-318 (1995)

10. Passat, N., Ronse, C., Baruthio, J., Armspach, J.-P., Bosc, M., Foucher, J.: Using multimodal MR data for segmentation and topology recovery of the cerebral superficial venous tree. In: Bebis, G., Boyle, R., Koracin, D., Parvin, B. (eds.) ISVC 2005. LNCS, vol. 3804, pp. 60-67. Springer, Heidelberg (2005) 